\title{
Working Under Grey Skies: Information Systems Development and Organizational Identity in a Post-merger Context
}

\author{
Dragos Vieru \\ TELUQ University Montreal \\ vieru.dragos@teluq.ca
}

\author{
Suzanne Rivard \\ HEC Montreal \\ suzanne.rivard@hec.ca
}

\begin{abstract}
This study pertains to the challenges encountered during the development of information systems aimed to support an organization created by the merger of previously independent entities. We draw from the concept of organizational identity and on the literature on knowledge sharing across boundaries to analyze three information systems development (ISD) projects in a large teaching healthcare centre resulting from the merger of five hospitals. We propose a multilevel model that suggests that the interaction of organizational identity and cross-boundary knowledge sharing may result in information systems with final functionality different from planned functionality. The model also suggests that organizational-level decisional events, such as the choice of integration approach between the merging entities, may influence how new information systems are developed at a group level, and how those organizational-level events, in turn, are shaped by group-level events.
\end{abstract}

\section{Introduction}

A merger is the result of a strategic decision aimed at increasing an organization's market shares, reduce its costs or create synergy [24]. A merger comprises three phases. The first two, pre-merger and merger decision, involve mostly strategic and financial analyses that identify the potential synergies of the planned merger. The third phase, post-merger integration (PMI), is the process of actual valuecreation that will hopefully materialize when the organizations are amalgamated [17].

There exist four generic PMI approaches [9]. Preservation refers to a situation where the old boundaries between the merging organizations remain intact. Absorption occurs when one of the firms imposes its work practices, norms and culture on the other parties. Symbiosis represents the integration approach in which the merging parties are gradually blended together by becoming increasingly interdependent and retaining the best parts of each organizational structures. Transformation reflects the situation in which organizations are integrated by developing totally new best work practices and a common organizational identity.

Despite the expected benefits of a merger, PMI is often plagued by problems such as employees' stress, dissatisfaction and resistance [11]. The literature suggests that these problems arise because of perceptions of inter-group differences [14], incompatible organizational cultures [25], feelings of exclusion [12], lack of organizational identification [30], and organizational identity ambiguity [5]. All these problems seem to refer to one core phenomenon: that during PMI, members of the new organization resulting from the union of previously independent entities may feel that their core organizational values and practices are endangered by the inculcation of a new organizational identity.

In addition to the merging of departments, processes and functions, a merger implies the development of new ISs that will span the boundaries of the previously independent organizations [33]. Research has shown, albeit not in a PMI context, that the success of ISD initiatives is highly dependent on effective collaboration and knowledge sharing among individuals in different professional communities [18, 28]. Given the difficulties that plague the PMI process, knowledge sharing during ISD initiatives is likely to be challenging since the actors involved abide by different local, social and cultural rules founded in different organizational contexts [27]. Although IS researchers stress the importance of "changes in IS strategy, IS structure, and systems supporting the combined IS and business units that allow them to function as a whole" [20: p.145], the ISD process has yet to be studied [32].

In this study, we aim to understand the challenges encountered when ISs are developed to support an organization that emerges from a PMI. To do this, we draw on the concept of organizational identity $[1,4]$ and on the literature on knowledge sharing across boundaries $[19,22]$ to analyze three ISD projects in a large teaching healthcare centre resulting from the merger of five hospitals. Our analysis suggests that 
divergent organizational identities, which with ISD team members' alternative interpretations of others' practices, norms and organizational symbols, coexist during PMI. These interpretations are reflected in the functionality of the IS under development in such way that in all three cases, the final functionality was different from the planned one.

We propose a multi-level process model that is enriched by incorporating two motors of change, teleological and dialectic [31]. The model suggests that the relationship between knowledge sharing and identity at the ISD group level has an effect on the integration approach decision at the organizational level.

\section{Theoretical background}

\subsection{Organizational identity}

Organizational identity constitutes mental representations of how organizational members define themselves as social group in terms of practices, norms, and values and understand themselves to be different from members of other organizations. At the individual level, it reflects the shared understanding of what the organizational norms, values and practices are [1]. At the organizational level, identity can be reified as an organizational asset, something that is durable or can be illustrated as a dynamic process, something that is continuously in a "becoming" phase formed by the amalgamation of the distinctive attributes of individuals [4]. Through continuous interaction, organizational members reconstruct their organizational identity through interpretive schemes in order to provide meaning to their experiences and practices as part of their membership to a specific organization [7]. The more an individual conceives of the self in terms of the membership of an organization, the more the individual's attitudes and behavior are governed by this organization membership [13].

We underline the central, distinctive and dynamic nature of organizational identity. The central aspect of identity is based on the core set of beliefs, values and norms rooted in the organizational mission that eventually justifies members' understandings of who they are [2]. The distinctive character is reflected by members' perceptions of the differences between their organization and others, usually competitors. According to Ashforth and Mael [2], the organizational members often magnify perceived differences and minimize similarities in order to provide justification of claims of superiority of their organization. Complex changes such as spin-offs and mergers trigger a process of change of the organizational identity [5]. Therefore, identity can also be understood as a dynamic process rather than just a static organizational asset [4]. Existing studies suggest that successful major organizational changes are linked to a change in organizational identity [e.g. 6, 10]. Overall these studies clearly point to the fact that the relationship between organizational identity and change is a dynamic process laden with uncertainty and that members' interpretive schemes have an impact on any attempt to change identity.

\subsection{Cross-boundary knowledge sharing in ISD}

We adopt a dynamic view of knowledge that emphasizes that knowledge is created and shared in social interactions [22]. Here, knowledge is defined as multi-faceted and complex, being situated and abstract, tacit and explicit. This approach advances that in order to understand how knowledge is created, articulated, disseminated and legitimized within organizations, knowledge should be considered as being an individual or group disposition embedded in organizational structures and in the social relationships evolving among the members of the same organization [22].

Sharing knowledge among people who are members of different organizations is difficult, since they usually do not share the same set of values, ideas, and interests. This makes tacit knowledge easily moved within groups based on similar identities in terms practices and norms, but difficult to be shared across different groups based on diverse organizational norms and values [3]. Sustained inter-organizational collaboration often leads to organizational boundaries that are based on different organizational identities or the choice of "who we are" [26]. Thus, knowledge bases within the same organizational boundaries of identity allow for efficient communication within the group at the expense of making communication and understanding difficult for outsiders.

\section{Methodology}

We adopted a theory building from case studies approach [8] with theoretical replication [21, 34]. We chose three retrospective cases (Table 1) representing three implemented ISD projects within one organization that was engaged in the process of post-merger integration. The selected organization was the Teaching Health Centre (THC - a fictional name), a Canadian tertiary care teaching institution. As shown in Table 1, similarities and variations of three characteristics of the cases were: type of business process enabled by the developed IS, planned integration approach, and final integration approach.

Consistent with an interpretive perspective on organizational identity [4], the organizational members 
constituted our main source of data. We conducted semi-structured interviews with the persons who had held key roles in each project. The interviews were supplemented by archival documents, which offered a source of triangulation for the themes that emerged from the interview data.

The interviewees were selected following a snowball sampling procedure. We interviewed department managers, IS professionals, project managers, and clinicians who participated in the development and implementation of the new ISs. A total of 33 interviews (Table 1) were performed for the three case studies. Interview questions focused on understanding, from the participant's standpoint, the history of the ISD projects collaboration practices, differences in identities, claims of relevant knowledge, differences in IS' functionalities between the initial and the go-live phases of the project, as well as personal implications of the merger. When no new information was revealed during interviews, data collection was terminated.

Archival sources included project documentation and minutes of project meetings and other meetings relevant to the merger (e.g., strategic planning sessions, management presentations, and communications planning).

Table 1. Selected cases

\begin{tabular}{|c|c|c|c|c|c|}
\hline & Business Process & $\begin{array}{c}\text { Planned Integration } \\
\text { Approach }\end{array}$ & $\begin{array}{c}\text { Final Integration } \\
\text { Approach }\end{array}$ & Timeline & $\begin{array}{c}\text { Number of } \\
\text { interviews }\end{array}$ \\
\hline Case 1 & $\begin{array}{c}\text { Patient Appointment } \\
\text { Scheduling }\end{array}$ & Transformation & $\begin{array}{c}\text { Mix of Preservation } \\
\text { and Transformation }\end{array}$ & $1997-2003$ & 9 \\
\hline Case 2 & Laboratory Services & Transformation & $\begin{array}{c}\text { Mix of Preservation } \\
\text { and Transformation }\end{array}$ & $2003-2006$ & 15 \\
\hline Case 3 & $\begin{array}{c}\text { Clinical Information } \\
\text { Management }\end{array}$ & Transformation & $\begin{array}{c}\text { Mix of Best-of-All } \\
\text { and Transformation }\end{array}$ & $2004-2008$ & 9 \\
\hline
\end{tabular}

The interview data were analyzed in an iterative process $[8,34]$. We cycled between data, emerging themes, and relevant literature to develop a deeper understanding of the dynamics of the ISD process. We first wrote the cases by synthesizing the interview transcripts. We then used the case narratives for withincase analysis and cross-case analyses. During withincase analysis, themes emerged from the data. During cross-case analysis, we compared in pairs to identify similarities and differences [21]. Coding was a twophase process. In Phase 1 we created a provisional "start list" of codes prior to the interviews. Most of the initial coding categories were based on the concepts of organizational identity [2], organizational boundary of identity [26] and on the four-ideal PMI approaches [9]. In Phase 2, the interview transcripts were introduced into a database, read carefully and relevant portions highlighted as "evidence". Occasionally, a segment of the transcript resulted in the refinement of an existing code or even the amalgamation of codes with similar meaning.

\section{Findings}

The THC is the outcome of the merger of five independent teaching hospitals. Even though the THC comprises five sites, from structural and decisional viewpoints there were only three main partners in the merger: two Adult hospitals, the Downtown and the Midtown, and the Paediatric hospital. The other two hospitals had historical collaborative ties to the Downtown hospital and provided specialized healthcare services. This organizational identity differentiation is reflected in the archival documentation and in the interviews, by the many references to differences, on one hand, between the Paediatric site and the Adult sites, and on the other hand between the two main Adult sites, the Downtown and the Midtown. The THC was created with the clear goal to provide 21st century health care by implementing a "best practices" business model for coordinating care.

\subsection{Case 1: The ambulatory appointment information system (AAIS)}

An AAIS is a patient scheduling system that enables clinical staff to manage a wide array of ambulatory care information, including appointments, registrations, attendances and waiting lists. Prior to the merger, the hospitals' ambulatory services used legacy mainframe-based systems that did not provide adequate appointment booking and patient related statistics to management. A project team was created to supervise the work of the developers from Omega, the company that was chosen to develop the AAIS. The project commenced in January 1997 and comprised two phases. In Phase 1, the members of the project team were the three managers of the ambulatory services at their sites (Downtown, Midtown and Paediatric), each using a different set of ambulatory practices based on 
pre-merger hospital-based norms. From the outset, teamwork was organized on a peer-based collaboration without a formal project manager. Team meetings were about exchanging clear information about the needs of each member's own department, in order to effectively negotiate and convince the others of the necessity of their demands for specific system features. The outcomes of these discussions were often a compromise representing shared understandings of the extent and uniqueness of everyone's site-based practices, norms and values. During Phase 1 the Paediatric manager was convinced that due to its procedural and clinical differences, the Paediatric site would never really be integrated with the rest of the THC and insisted to have the new system's functionality be compatible with the old Paediatric site's norms. In the spring of 1998, the upper management decided to implement the new AAIS only at the Paediatric site and evaluate of the system a year later. Phase 1 of the project was completed at the end of 1999.

In early 2000 , the THC upper management realized that after almost two years of PMI, not only the Paediatric site had kept their clinical independence, but also within the Adult sites, with some notable exceptions like the Radiology and the Emergency departments, the administrative staff was preserving their old practices and norms. In this context, upper management realized that in order to successfully implement a unique set of administrative ambulatory practices, they had to develop and deploy a multi-site version of the AAIS at the Adult sites. Phase 2 of the project commenced with a different team structure. While the two Adult site managers were still present, three IS professionals were added to the team (a project manager and two IS specialists). In 2003, the implementation of the AAIS at the Adult sites was completed. While the pre-merger THC strategic plan stressed the importance to implement a common set of administrative practices, at the end of AAIS project, the ambulatory services were presenting two different sets of practice: one that preserved its old norms (Paediatric) and another, at the Adult sites, based on a common set of best practices. Thus, the resulted AAIS functionality reflected a blend of preservation (Paediatric site) and transformation (Adult sites) and was different from the planned configuration (transformation).

4.1.1. Within-case analysis. Theme 1: Us-versus-them. At the outset of the project, there were three site-based set of practices: the Midtown site, the Downtown site and the Paediatric site.

"There were a lot of procedural differences in terms of how the clerical tasks were done. It was a significant amount of difference between the sites" (Midtown manager)

In these conditions knowledge sharing among the team members was never easy during the ISD process. Team members realized that they would have to find ways to mitigate the different understandings of how the future system functionality should look like. These viewpoints were reflecting the pre-merger organizational norms and values, and obvious consensus had to be reached. In Phase 1, Paediatric manager described her site as being completely different from the other sites of the THC in terms of administrative work and refuted any idea of integration. In her interview, she states:

"I have to say, we being the Paediatric, probably influenced a lot because the whole project actually came from a needs analysis that we had submitted [...]. They moved Orthopaedics from the Downtown and centralized it at the Midtown. I think they've redone some of their management structure in terms of that. But that didn't affect us." The evidence confirms the fact that the practices of symbolic "us-versus-them" were necessary for the Paediatric manager to represent her organization as being unique. In our opinion, the Paediatric manager engaged in this symbolic discourse to reinforce her perceptions of the enduring and central nature of the pre-merger organizational identity and justify the way the system was configured at the end of Phase 1 .

In Phase 2, the enduring nature of the old identities was still obvious. One of the IS specialists involved in the project remembers that, when she started visiting the clinics with the Midtown manager and Downtown manager, she would hear them saying all the time:

"You are implementing the system at the Midtown.

You're implementing it at the Downtown... we do things differently at the hospitals. We need to be distinct."

Theme 2: Enduring nature of the old organizational identities. The representatives of the three main merging organizations described the existence of sitespecific understandings of the rationale for the different norms and practices and the meanings that legitimized the continuity of their application during the PMI process. On one hand, the Paediatric manager was focused on her organizational-based set of practices and was convinced that:

"I had fought for getting it for Paediatric, because there's nothing worse than implementing a new system and losing functionality of the things you had before."

On the other hand, the two Adult site managers understood that despite different site idiosyncrasies, at least the Adult sites need to find ways to blend in their practices and adopt a common set of best practices. 
[On the Adult sites] "At the beginning it was sort of they each keep their own practices and it's only over the course of time that the wall has got beaten down a bit... That more and more is becoming a case of blending... As far as I'm aware, the Paediatric is standalone... They do what they do inside their black box." (Midtown manager)

\subsection{Case 2: The laboratory information system (LIS)}

In 2002, upper management decided to implement a unique across the sites new LIS (technological platform provided by company Sigma), which would improve the quality of patient care by providing comprehensive overall functionality, accessibility to data throughout the THC. In general, the role of an LIS in a hospital is to automate laboratory clinical, financial and managerial processes. At the outset of the project, THC upper management proposed guidelines for the standardization of the practices of the three main laboratories. The entry of lab requests involves typing, or scanning (where barcodes are used) of the laboratory number, and entering the patient identification that gives a destination (hospital department/physician) for results to go. Even though this description of a typical lab workflow seems to be straightforward, each of the three lab services at the THC was using different workflows and different legacy ISs. During Phase 1 of the project, the lab clinicians from the three sites struggled to find common grounds for establishing unique workflows tried to accommodate as many old procedures and workflows as the new LIS would accommodate.

At the end of 2004, Sigma advised THC that it would provide new version of their LIS platform that will force the LIS team members to start from scratch the process of building the database of the system. This was seen as an opportunity by the upper management to put pressure on clinicians to reach an agreement on common lab procedures. Phase 2 of the project concluded in September 2005, when the new LIS based on common lab practices was put into production at the Downtown site, flowed by Midtown and Paediatric sites in February 2006. While the initial design was based on Sigma's approach to implement industry best practice standards, the final configuration was based on the team members' understandings of best practices as being a blend of industry standards and local contingencies. Therefore, the resulted LIS functionality reflected a mix of transformation (Adult sites) and preservation (Paediatric site) PMI approaches.

4.2.1. Within-case analysis. Theme 1: Us-versus-them. At the outset of the Phase 1, there were three-site based sets of lab procedures: the Midtown site, the Downtown site and the Paediatric site. One of the team members remembers that:

"We had Downtown working one way, Midtown working another way, Paediatric working a different way. That was like 'Joe' works at this bench. 'Jim' works on the same bench; he's going to work on what he thinks on that bench. You take those two people that have different visions of doing the same work and multiply it by three sites." (Lab technician Downtown)

In these conditions, sharing knowledge was not possible before team members understood the differences between the practices of the three lab services at the end of Phase 1. In Phase 2, while trying to negotiate common procedures, team members from the Adult sites engaged in discourses that overstated the differences in practices and norms between their sites and tried to convince the other members how much better one lab was over the another ones:

"The Midtown was always a more efficient lab of the three sites. The Downtown was very specialized in all kinds of esoteric testing. They were not as efficient as the Midtown was." (Lab services Director Midtown)

"When you talk to the Midtown people they will tell you they are efficient than the Downtown and at the Downtown the staff don't know what they are doing, etc." (Pathologist Downtown)

The evidence points to the fact that these discourses of "us-versus-them" were part of the ongoing "war" between the two Adult sites during the two phases of the project. This situation shed light on the existence of a continuing struggle to impose one organization identity as being dominant over and against the other competing alternative.

Theme 2: Enduring nature of the old organizational identities. Some of the team members saw the implementation of the new LIS as a means to reify their loss of organizational identity. Some of the excerpts of the interviews reflect this fact:

"They didn't give us a chance to mourn [...]. We were losing the identity that we had as standalone areas" (Lab technician Downtown); "The overall consequence was the loss of institutional identity" (Physician Midtown)

To resist the emergence of a new identity common across the site boundaries, team members tried to perpetuate the old organizational identities, even though in some cases this was counterproductive for everybody at the THC.

"The culture within THC has always been five hospitals for them, and it's very difficult to be able, even at the level of directors, to make them understand that when we compete against [other 
healthcare institutions] we need to work together." (Microbiologist Midtown)

Each pre-merger organizational identity was based on common beliefs about the value of their contextual practices, of what was "at stake". Thus, the evidence suggests that when members showed little interest in the others' "stakes", the project didn't advance well and eventually stalled (Phase 1). Only when pressured by the upper management (Phase 2), had the team members to learn to acknowledge and understand the others' rules and values that eventually lead them to realize that trade-offs were available for them.

\subsection{Case 3: The clinical display (CD - CIS)}

In the summer of 2004, the THC decided to implement in several major phases a Clinical Information System (CIS) by signing a contract of collaboration with Delta, a supplier of CIS solutions. A CIS is the most complex IS in terms of patient data management and it offers one-stop access to patient information by centralizing all electronically available clinical data. Delta CIS offered in Phase 1 a Clinical Results Display that provided a unique "smart summarization" in a series of screens that display patient demographics and clinical results. Our study examined only this phase of the project due to the fact that the other phases were still in progress at the time of writing this case. Phase 1 was completed in December 2008. Basically, the Clinical Display was supposed to bring information, scattered across the THC sites, to one central access point in front of any THC caregiver.

Even though the assessment of the requirements and the configuration of the interfaces between the ancillary systems and the CIS seemed to be a straightforward process, soon the team members realized that, due to the differences in procedures and norms between the three main sites of the THC, they would have to clearly evaluate the system's limits of configurability versus clinicians' expectations. At the outset of the project it was anticipated that a first draft of the design of the Clinical Display would be ready by the end of 2004 and a production version would start being implemented in 3 pilot departments each at each of the three main sites of the THC by mid-2005. However, budgetary constraints triggered important delays. Finally, the pilot test was ready to start in May 2006. In September 2006, the conclusions regarding the outcomes of the Pilot test were presented to upper management. A list of issues and the propositions of how to solve these issues were advanced. The team members had to strike a compromise between the needs of the respective department and the level of configurability of CIS. On one hand, the fact that now the nurses had to work with only one system instead of several ancillary systems constituted a major change in their workflow. On the other hand, the new IS was providing to the physicians a single point of access to patient information, a sort of "best of all worlds" regardless of physicians' physical working place across the THC sites. In conclusion, our analysis found a mix of transformation (for nurses) and symbiosis (for the physicians) for the resulted post-merger integration approach compared to a transformation level for the planned IS configuration.

4.3.1. Within-case analysis. Theme 1: Us-versus-them. Even though after 7 years of PMI, at the outset of this project there were still three distinct sets of practices: the Midtown, the Downtown, and the Paediatric. A nurse from the Paediatric site illustrates this situation:

"It was clear that we were working in different cultures [Adult sites and Paediatric site] because the three sites had different workload systems, so the way things were functioning and working with it was different"

Data from the interviews show that team members had to first establish common meanings about practices before being were able to deal with the different interests among the team members regarding the Clinical Display configuration. Mid-way through the ISD process, the negotiation of trade-offs among the members generated frustration especially when the management had to decide which departments would be designated as beta pilot environments. Everybody wanted to have their department designated as a show case for the new technology within the THC. The perception of "we-versus-them" was still present. According to a physician representing the Downtown,

"The Midtown physically is vertically aligned and is very much army driven in terms of hierarchical structure. So decision-making happens at the very top and people at the bottom really do not speak to anybody outside their silo. Whereas, at the Downtown physically we're almost like a cooperative and that's the way organizationally and personally we've been interacting."

The evidence suggests that even though officially abolished, the three main pre-merger hospitals continued to exist in the minds of the individuals who clearly delineated boundaries around them: people at the Paediatric site "get things done", Midtown site has an "army structure", and Downtown is like a "userfriendly computer".

Theme 2: Enduring nature of the old organizational identities. The team members recognized that even after all these years of PMI, each site still has a clear recognizable set of values and norms that will not easily be erased. 
"Yes there's a Downtown and there's a Midtown culture. So you need to know what's going on within your thing." (Nurse Downtown); "The Paediatric, from the beginning of the merger they had a sense that they're different, which is true." (Nurse Midtown)

The evidence suggests that in this case, the team members acknowledged and understood from the beginning what is "at stake" for the others which made the ISD process to advance as planned.

\subsection{Cross-case analysis}

Theme1: Us-versus-them - Our cross-case analysis revealed that in all three cases some of the team members engaged in symbolic discourses of "usversus-them" that were part of an ongoing process of justification of why each site was unique and had different needs than the other sites during the ISD processes. Even though, the merger was announced in 1997, the boundaries around the pre-merger hospitals continued to exist in the minds of the individuals during all three ISD processes. Thus, the ever-present competitiveness among the three main THC sites and the perpetuation of the pre-merger organizational identities made decisions at the THC organization level to be acknowledged but differently applied at the department or group level.

Our analysis suggests that the symbolic discourses of "us-versus-them" have a relationship with the length and the outcomes of the three ISD processes. While these discourses were consistent throughout the three ISD processes their effect diminished over time. In Case 1, the "us-versus-them" engaged by the Paediatric manager influenced the outcomes of the project and made it last almost 7 years. In Case 2 this had as a result a two-year delay for delivery and had as outcome a mix of transformation and preservation. In Case 3 the same type of discourses was still present, however, this time the evidence suggests that only the outcome was affected (mix of transformation and symbiosis). Taking into consideration the above argumentation we propose a first research proposition:

P1: Dialectical argumentation of "us-versus-them" will affect the outcomes of and will lengthen the ISD project processes. Their effect will be stronger (towards a preservation of practices) for processes initiated at the beginning of the PMI phase than for processes initiated later.

Theme 2: Enduring nature of the old organizational identities - The cross-case analysis revealed one main observation: the interviewees considered that there was a rationale for each different set of practices. For example, in Case 2, a lab technician considered that
Downtown practices were based on pre-merger organizational identity-related set of values. He saw the new LIS implementation as being an occasion for the members of his field to "mourn" the old ways of doing their jobs. Overall, the interviewees' comments reflect the existence of separate contextual meanings and organizational symbols at each site. The individuals, as members of the same pre-merger organization, shared an identity, which was based on an agreement on what was at "stake" in each organization. Thus, in all three cases effective knowledge sharing happened only after individuals started to acknowledge and understand that different "stakes" needed to be taken into consideration during the process of negotiation of common interests. For instance, in the first two cases when the team members had no interest understanding the other different sets of norms and values, the project had unexpected outcomes (Case 1 - Phase 1) or came to a standstill (Case 2 - Phase I). In Case 3 the members acknowledged from the outset what was "at stake" for everyone which made the ISD process to advance as planned. Based on the above argumentation, we advance a second research proposition:

P2: Acknowledging and understanding the reason for each set of different organizational identitybased norms and values will enable team members to effectively share knowledge during ISD in PMI.

\section{A multilevel model of ISD in PMI}

Our model is based on two key premises. First, it conceptualizes organizational identity boundaries among to-be-merged organizations as being based on shared values, practices, and norms. Second, it espouses the idea that ISs are characterized by a "lack in completeness of being" [15] during their development, which means that ISs under development have the capacity to continuously unfold, as they are not static, fixed, or given. Thus, during the ISD process through knowledge sharing, the ISs are continuously defined and their functionality continuously evolves during their development.

We espouse the view that organizations must be considered as being multilevel phenomena [29] and that theories of change should take into consideration how "processes at different levels affect each other" [23]. Important change processes in organizations, such as PMI, can be explained alternatively or complementarily over time by four different theories of change or "motors": life cycle, teleology, dialectic and evolutionary [31]. The multilevel process model developed in this study employs a dual-motor perspective. It provides an explanation of how organizational-level decision events, such as the choice of the integration approach, impact on how the 
functionality of new ISs will be designed and developed at a group level, and how those organizational-level events, in turn, are shaped by the group-level events and effects. We consider the process of a post-merger ISD project from a dual-motor perspective: teleological and dialectical.

The model, illustrated in Figure 1, is based on a number of assumptions. The first assumption is that change is driven by the actions of organizational members, usually managers, who try to create a new organizational form. The second assumption is that these individuals are rational, as they are assumed to be in the traditional teleological models of change. These rational individuals plan the implementation stages in order for organizational strategic goals to be met. As per the third assumption, organizations are complex entities that are comprised of goal-driven individuals whose personal agendas might be incompatible with the organization's (e.g., such as the physicians and nurses in Lapointe and Rivard's [16] description of user resistance to a new medical IS). From this, it is inferred that the mechanism for driving change is dialectical because change is the product of the interplay between opposing forces.

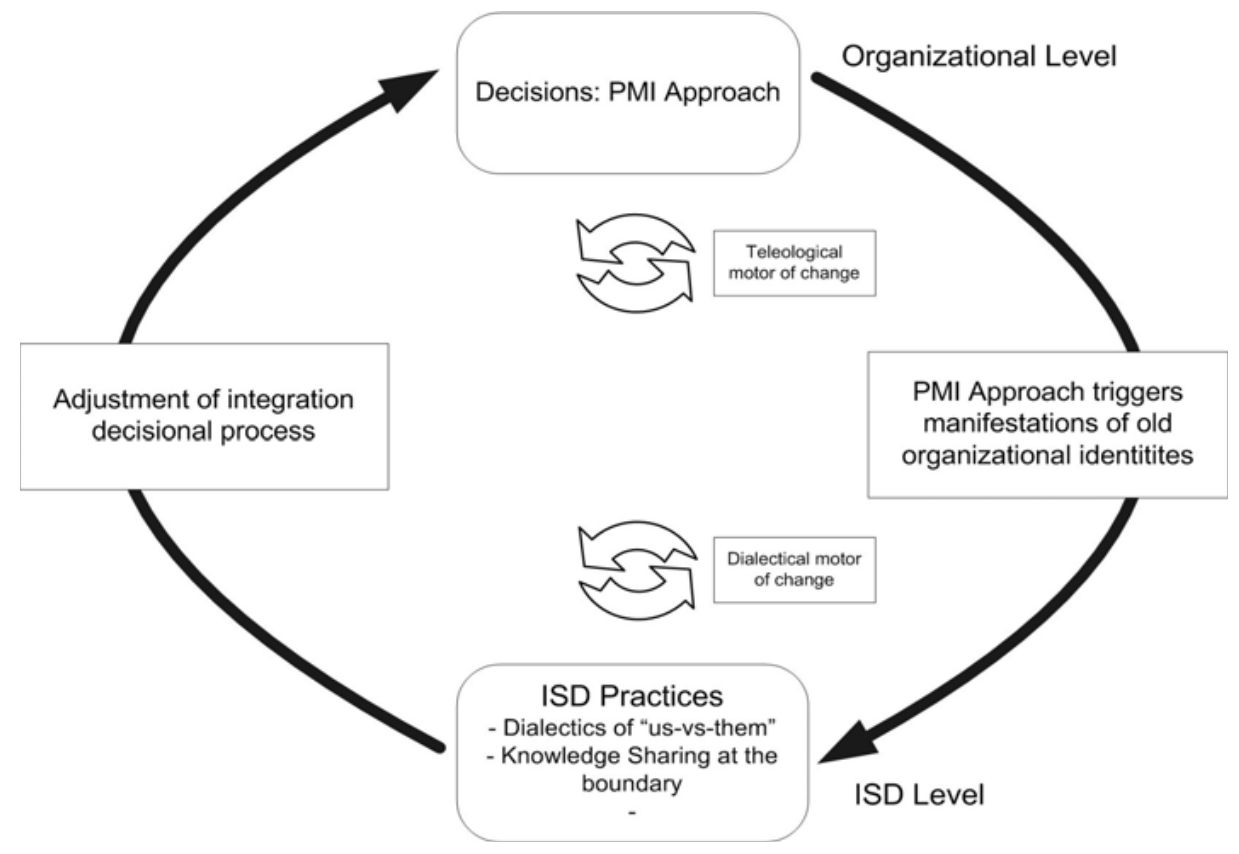

Figure 1. Post-merger ISD process model

Figure 1 illustrates the operation of both teleological and dialectical motors across the process of knowledge sharing at the organizational identity boundary during a post-merger ISD. The model operates at two levels: the organization (teleological motor) and the ISD project (dialectical motor). First, we posit that a teleological motor operates at the organizational level where upper management decides to implement ISs that would reflect work practices of a specific integration approach among the structures of the merging organizations. The integration approach decision will reveal existing more or less salient premerger organizational identity boundaries between members involved in an ISD. At the ISD level, we conjecture that team members will try to use their premerger organizational identity to influence the design of the IS functionality (dialectical motor). Thus, the initial functional design of the IS that reflects practices related to a specific PMI approach may be different from the final functionality at the end of the ISD process. The resulting dialectic leads to an iterative process of knowledge sharing and negotiation of common interests at the boundary and to a change/adaptation in the decisional process regarding the integration approach at the organizational level.

In Case 1, the management of the new merger organization decided to acquire the AAIS (Phase I teleological motor). The decision to implement new best practices triggered discourses of "us-versus-them" (Phase 1 - Paediatric manager) in order to impose boundary conservation around the Paediatric site (dialectical motor). At the end of Phase 1, management accepted to install the system only at the Paediatric site (decisional adjustment). At the beginning of Phase 2, management decided that the system needs to be installed at the Adult sites to enable common best practices (teleological motor). The team members were able to negotiate common interests and develop an IS that reflected new best practices (dialectical motor). The outcome of the ISD process was an IS reflecting 
two different sets of practices (decisional adjustment mix of transformation and preservation). In Case 2, management decided to implement a common LIS (teleological motor). Phase 1 is characterized by the agents' struggle to defend their old lab procedures (dialectical motor). At the end of Phase 1, management decided to stop the project and re-assess the situation (decisional adjustment). At the outset of Phase 2, the management decided that the system must reflect common new practices (teleological motor). In Phase 2 , some members engaged in discourses of "us-versusthem" but they also negotiated trade-offs (dialectical motor) to advance the project. The outcome of the ISD was an IS that enabled a main set lab practices and accommodated the particularities of the Paediatric site (decisional adjustment - mix of transformation and preservation). In Case 3, management took the decision to have a common CIS (teleological motor). The team members identified common understandings about their old organizational identity-based practices and negotiate shared interests (dialectical motor). In the end management accepted to implement an IS that was reflecting a new set of practices for the nurses, and "best-of-all" practices for the physicians (decisional adjustment - mix of transformation and preservation).

\section{Implications and future research}

We developed a multilevel process model that suggests micro- and macro-levels of analysis can be simultaneously studied. The data analysis revealed that while the planned post-merger integration approach was a transformation, the outcomes of the three projects suggest a mix of preservation and transformation for Cases 1 and 2 and a mix of symbiosis and transformation for Case 3 .

Our study makes a number of contributions. In terms of practical implications, this research emphasizes that while it is paramount to develop and implement ISs with functionalities that enable postmerger practices, management would be in a better position to make a decision regarding the integration approach if it understood why similar business processes were performed differently in the previously independent organizations. In terms of contributions to IS research, first, by using the concept of organizational identity we were able to see that the final functionality of the three developed IS reflected individuals' understandings of the others' norms, values and organizational symbols. Second, we developed a dual-motor process model that provides an explanation of how organizational-level decisional events, such as the choice of level of PMI, impact on how the functionality of new ISs will be designed and developed at a group level, and how those organizational-level events, in turn, are shaped by the group-level events and effects.

THC was a unique setting in many respects and it would be fruitful that future research continues building the theory developed in this study based on data from other PMI settings in different industries. The extant literature on PMI indicates that mergers suffer a high failure rate and one of the main reasons is ineffective collaboration [35]. The fact the transformation integration approach adopted at THC exacerbated the differences in pre-merger organizational identities should constitute food for thought for researchers assessing factors that impact merger outcomes.

\section{References}

[1] Albert, S. and Whetten, D. "Organizational identity," In L. Cummings and B. Staw (eds), Research in Organizational Behavior (70), pp. 263-295, Greenwich, CT: JAI Press, 1985.

[2] Ashforth, B.E. and Mael, F.A. "Organizational identity and strategy as a context for the individual", Advances in Strategic Management (13), 1996, pp. 17-62.

[3] Brown, J.S. and Duguid, P. "Knowledge and Organization: A Social-practice Perspective," Organization Science (12:2), March/April 2001, pp.198-213.

[4] Clark, M.S., Gioia, D., Ketchen Jr, D.J. and Thomas, J. "Transitional Identity as a Facilitator of Organizational Identity Change during a Merger," Administrative Science Quarterly (55:3), 2010, pp.397-428.

[5] Corley, K. and Gioia, D. "Identity ambiguity and change in the wake of a corporate spin-off," Administrative Science Quarterly (49), 2004, pp.173-208.

[6] Dutton, J. and Dukerich, J. "Keeping an Eye on the Mirror: Image and Identity in Organizational Adaptation," Academy of Management Journal, (34), 1991, pp 517- 554.

[7] Dutton, J., Dukerich, J. and Harquail, C. "Organizational images and member identification," Administrative Science Quarterly (39), 1994, pp.239-263.

[8] Eisenhardt, K. M. "Building Theories from Case Study Research," Academy of Management Review (14:4), 1989, pp. 532-550.

[9] Ellis, K. "Managing the Acquisition Process: Do Differences Actually Exist across Integration Approaches", in Pablo, A.L. and Javidan, M. (eds.), Mergers and Acquisitions: Creating Integrative Knowledge, Blackwell, Oxford, 2004, pp.113-132.

[10] Gioia, D. A. "From individual to organizational identity," in D. A. Whetten and P. C. Godfrey (eds.), Identity 
in Organizations: Building Theory through Conversations, Thousand Oaks, CA: Sage, 1998, pp.17-32.

[11] Greenwood, R., Hinings, C.R. and Brown J. "Merging Professional Service Firms," Organization Science (5:2), May 1994, pp.239-257.

[12] Harwood, I. and Ashleigh, M. "The Impact of Trust and Confidentiality on Strategic Organizational Change Programmes: A Case Study of Post-acquisition Integration," Strategic Change (14:2), 2005, pp.63-75.

[13] Hogg, M. A. and Terry, D. J. "Social contextual influences on attitude-behavior correspondence, attitude change, and persuasion," in D. J. Terry and M. A. Hogg (Eds.), Attitudes, behavior, and social context: The role of norms and group membership, Mahwah, NJ: Erlbaum, 2000, pp.1-9.

[14] Jetten, J., O'Brien, A and Trindall, N. "Changing Identity: Predicting Adjustment to Organizational Restructure as a Function of Subgroup and Superordinate Identification," British Journal of Social Psychology (41:2), 2002, pp.281297.

[15] Knorr-Cetina, K. "Objectual Practice", in Schatzki, T.R., Knorr-Cetina, K. and von Sevigny, E. (Eds.), The Practice Turn in Contemporary Theory, Routledge, London, 2001, pp.175-188.

[16] Lapointe, L. and Rivard, S. "A Model of Resistance to Information Technology Implementation," MIS Quarterly (29:3), 2005, pp.461-492.

[17] Larsson, R. and Finkelstein, S. "Integrating Strategic, Organizational, and Human Resource Perspectives on Mergers and Acquisitions: A Case Survey of Synergy Realization," Organization Science (10:1), 1999, pp.1-26.

[18] Levina, N. and Vaast, E. "The Emergence of Boundary Spanning Competence in Practice: Implications for Implementation and Use of Information Systems," MIS Quarterly (29:2), 2005, pp.335-369.

[19] Levina, N. and Vaast, E. "Innovating or Doing as Told? Status Differences and Overlapping Boundaries in Offshore Collaboration," MIS Quarterly (32:2), 2008, pp.307-329.

[20] Mehta, M. and Hirschheim, R. "Strategic Alignment in Mergers and Acquisitions: Theorizing IS Integration Decision Making," Journal of the Association for Information Systems (8:3), 2007, pp. 143-174.

[21] Miles, M. B. and Huberman, A.M. Qualitative Data Analysis, Sage, Thousand Oaks, 1994.

[22] Orlikowski, W.J. "Knowing in Practice: Enacting a Collective Capability in Distributed Organizing," Organization Science (13:3), May/June 2002, pp. 249-273.
[23] Poole, M.S. and Van de Ven, A. "Theories of Organizational Change and Innovation Processes", in Poole, M.S. and Van de Ven, A.H. (Eds.), Handbook of Organizational Change and Innovation, Oxford University Press, New York, 2004, pp.374-397.

[24] Puranam, P., Singh H. and Chaudhuri, S. "Integrating Acquired Capabilities: When Structural Integration is (Un)necessary," Organization Science, (20:2), 2009, pp.313328.

[25] Riad, S. "The Power of 'Organizational Culture' as a Discursive Formation in Merger Integration," Organization Studies (26:10), 2005, pp.1529-1554.

[26] Santos, F. and Eisenhardt, K. "Organizational Boundaries and Theories of Organization," Organization Science (16:5), 2005, pp.491-411.

[27] Schweizer, L. "Organizational Integration of Acquired Biotechnology Companies into Pharmaceutical Companies: The Need for A hybrid Approach," Academy of Management Journal (48:6), 2005, pp.1051-1074.

[28] Suchman, L. "Practice-Based Design of Information Systems: Notes from the Hyper-developed World," The Information Society (18), 2002, pp.139-144.

[29] Tsoukas, H. and Chia, R. "On Organizational Becoming: Rethinking Organizational Change", Organization Science (13:5), 2002, pp.567-582

[30] van Dick, R., Ullrich, J. and Tissington, P.A. "Working Under a Black Cloud: How to Sustain Organizational Identification after a Merger," British Journal of Management (17), 2006, pp.S69-S79.

[31] Van de Ven, A.H. and Poole, M.S. "Explaining Development and Change in Organizations," The Academy of Management Review (20:3), 1995, pp.510-540.

[32] Vieru, D. and Rivard, S. "Mind the Gap: Positioning Information Systems/Information Technology within the Process of Post-merger Integration," Proceedings of the Thirteenth Americas Conference on Information Systems, Keystone, Colorado, 2007.

[33] Wijnhoven, F.S., Stegwee, T.R. and Fa, R.T.A. "Postmerger IT Integration Strategies: An IT Alignment Perspective," The Journal of Strategic Information Systems (15:1), 2006, pp.5-28.

[34] Yin, K.R. Case Study Research Design and Methods, Sage Publications, 2003.

[35] Yoo, Y, Lyytinen, K and Heo, D. "Closing the Gap: Towards a Process Model of Post-merger Knowledge Sharing", Information Systems Journal (17), 2007, pp.321347. 